\title{
Wireless Brain-Robot Interface: User Perception and Performance Assessment of Spinal Cord Injury Patients
}

\author{
Alkinoos Athanasiou, ${ }^{1,2}$ George Arfaras, ${ }^{1}$ Niki Pandria, ${ }^{1}$ Ioannis Xygonakis, ${ }^{1}$ \\ Nicolas Foroglou, ${ }^{2}$ Alexander Astaras, ${ }^{1,3}$ and Panagiotis D. Bamidis ${ }^{1}$ \\ ${ }^{1}$ Biomedical Electronics Robotics \& Devices (BERD) Group, Lab of Medical Physics, School of Medicine, Faculty of Health Sciences, \\ Aristotle University of Thessaloniki (AUTH), 54124 Thessaloniki, Greece \\ ${ }^{2} 1$ st Department of Neurosurgery, "AHEPA” University General Hospital, Aristotle University of Thessaloniki (AUTH), \\ 54636 Thessaloniki, Greece \\ ${ }^{3}$ Robotics Laboratory, Computer Science Department, American College of Thessaloniki (ACT), 55535 Thessaloniki, Greece
}

Correspondence should be addressed to Alkinoos Athanasiou; athalkinoos@auth.gr

Received 25 August 2017; Accepted 10 December 2017; Published 31 December 2017

Academic Editor: Kyriaki Kalimeri

Copyright (C) 2017 Alkinoos Athanasiou et al. This is an open access article distributed under the Creative Commons Attribution License, which permits unrestricted use, distribution, and reproduction in any medium, provided the original work is properly cited.

\begin{abstract}
Patients suffering from life-changing disability due to Spinal Cord Injury (SCI) increasingly benefit from assistive robotics technology. The field of brain-computer interfaces (BCIs) has started to develop mature assistive applications for those patients. Nonetheless, noninvasive BCIs still lack accurate control of external devices along several degrees of freedom (DoFs). Unobtrusiveness, portability, and simplicity should not be sacrificed in favor of complex performance and user acceptance should be a key aim among future technological directions. In our study 10 subjects with SCI (one complete) and 10 healthy controls were recruited. In a single session they operated two anthropomorphic 8-DoF robotic arms via wireless commercial BCI, using kinesthetic motor imagery to perform 32 different upper extremity movements. Training skill and BCI control performance were analyzed with regard to demographics, neurological condition, independence, imagery capacity, psychometric evaluation, and user perception. Healthy controls, SCI subgroup with positive neurological outcome, and SCI subgroup with cervical injuries performed better in BCI control. User perception of the robot did not differ between SCI and healthy groups. SCI subgroup with negative outcome rated Anthropomorphism higher. Multi-DoF robotics control is possible by patients through commercial wireless BCI. Multiple sessions and tailored BCI algorithms are needed to improve performance.
\end{abstract}

\section{Introduction}

Spinal Cord Injury (SCI) is a potentially life-changing condition, causing permanent disability, compromising the victim's physical and psychological well-being and impacting their close environment as well. Brain-computer interfaces (BCIs) are rapidly developing into a field-changing technology for those patients, not only replacing motor functions $[1,2]$ but even promising to alter the long-term outcome of the condition $[3,4]$. Wireless invasive (implantable) neural recording is also an important development $[5,6]$, especially considering that SCI has become the research target of several assistive technologies [7] including functional electrical stimulation [8] and robotics for neurorehabilitation [9].
On the other hand, noninvasive BCI technology is dominated by the EEG modality, which has great temporal but low spatial resolution. EEG can detect rapid dynamics of the brain but lacks source estimation. Consequently, a major limitation of noninvasive $\mathrm{BCI}$ is the low signal to noise ratio (SNR) which can be partially attributed to volume conduction effect $[10,11]$. Since the EEG signal measured at the scalp is the superposition of all electrical signals, including those generated by the cortex, discriminating brain activity from artifacts and noise can be technically difficult.

In addition to these neurophysiological limitations, a major drawback of traditional noninvasive BCIs is inconvenience. The subject's movement is typically constrained by wires connecting the EEG electrode cap to the data 
acquisition unit, while the entire setup of wet electrodes entails a time-consuming procedure. From the hardware perspective, the main challenges of noninvasive BCI technology are portability and ease-of-use, rendering the wireless dryelectrode cap a promising solution. Lessons could be derived from the-otherwise immobile-MEG modality that boasts contactless sensors and reduced preparation time [13]. In terms of performance, the main challenge for noninvasive BCI technology is to accurately control external devices (multijoint robotic arms, drones, wheelchairs, etc.) that can move along several degrees of freedom (DoFs), a task which is necessary for performing complex operations in the physical world. Such a feat requires highly accurate decoding algorithms, able to discriminate multiple classes of Motor Imagery (MI) under the limitations of low SNR and spatial resolution. It has been demonstrated that the basic operation of a robotic arm which fits the aforementioned description is possible using noninvasive BCI [14].

Continuous advancements in electronics, from solidstate transistors in the 1940s, integration of sensors and powerful digital microprocessors in the 1970s, and other developments in the following decades, such as switching frequency, increased computing power, and programming flexibility, decreased fabrication cost and power consumption and steadily led to lighter and more agile, responsive, and computationally complex robots. The demand in the fields of Medical Rehabilitation and Assistive Technologies (AT) leads to the first combined medical applications for paralysis and stroke patients: robotic sleeves for assisted living, powered orthotics, and even some initial attempts at robotic manipulation arms $[15,16]$. This nascent field of Rehabilitation Robotics started taking shape in the 1970s and 1980s, gradually emphasizing novel robotic manipulator designs $[17,18]$ and attempts to adapt existing industrial robotic manipulators [19].

Unobtrusiveness, portability, and mobility are clear advantages for AT and Medical Robotics. Even though affordable, power-autonomous, full-body robotic exoskeletons are yet to materialize, disabled patients now have a variety of product options, some commercially available: manipulator arms mounted on robotic wheelchairs [20, 21], dexterous robotic prosthetics which can acquire their control signals directly from the patient's nervous system $[1,22]$, and adaptations of aforementioned BCI technology specifically for robotic rehabilitation [23-25]. Unfortunately, research in optimal interfaces for Human-Robot Interaction (HRI) is often overlooked in medical robotics development [12], with a potentially significant impact on user acceptance and the validation success rate for new technologies [26]. User perception and overall satisfaction with a robot's technological interface has equal importance to hardware/software design and quality standards, something that holds particularly true in robotic-assisted rehabilitation. The need for patient's immersive experience and willingness to collaborate with the robot, physiotherapist, and supervising physician should meaningfully alter the criteria of success of a robot [27]. For instance, acceptance of an external machine as part of one's own body schema can significantly impact the rehabilitation process and should also be taken into account $[15,28]$.
In our previous work we have presented our progress towards developing 8-DoF anthropomorphic robotic arms, controlled by wireless off-the-shelf BCI, for AT and rehabilitation applications [12]. We have accounted for development of the robotic arms and electronics, for implementation of the BCI control module, and we have presented pilot experimental applications of the Brain-Robot Interface (BRI) on healthy and disabled individuals $[29,30]$.

In the remainder of this paper we present an elaborate user-assessment study of our wireless BRI by subjects with SCI and healthy individuals [31]. We focus not only on performance assessment during multiple movements BCI control, but on user perception of the assistive technology as well, analyzed with regard to their neurological condition, independence, imagery capacity, and psychometric evaluation. In Materials and Methods, we briefly present the BRI and we explain the experimental setup and statistical analysis of our collected data, which we then present in Results. In Discussion we attempt to interpret our results, comment on the strengths, and underline the limitations of our approach. We also discuss planned technological development in the direction of robotics, BCI module, signal analysis, and further experiments, as well as the challenges we are yet to meet.

\section{Materials and Methods}

2.1. The Brain-Robotic Arms Interface. As both the interface's and robotics' development have been thoroughly described before [12, 32], we will briefly explain the system's technical characteristics here, emphasizing wireless capabilities. The Emotiv EPOC is an easily applied wireless 14-saline feltelectrode EEG system (Emotiv, USA), capable of detecting brain activity at a bandwidth of $0.2-43 \mathrm{~Hz}$, employing digital notch filters at $50 \mathrm{~Hz}$ and $60 \mathrm{~Hz}$ and built-in digital 5th order Sinc filter. Meanwhile, due to being commercial class, it costs significantly less and also is less obtrusive, simple to use, and portable compared to traditional medical EEG devices. Its technical characteristics are also on par with the designated use case, so our team used this device for the development of the BCI modality. The wireless chipset transmits at a proprietary ultra-low energy (ULE) $2.4 \mathrm{Ghz}$ Bluetooth Smart to a USB dongle connected to a dedicated computing laptop. Internal sampling rate of the electrodes is at $2 \mathrm{kHz}$ (sequential sampling and single analog to digital conversion) with 16-bit resolution and signal sampling rate of $128 \mathrm{~Hz}$ transmitted. Using the Cognitiv suite a resting state and up to four different mental commands are classified (using proprietary BCI algorithm) and then are appended to key button input through the Emokey software. The input then accesses a control MATLAB script corresponding to movement commands for the robotic arms (Figure 1). Movement coordinates are subsequently transmitted to the robotic arms system through a serial port with a Baud Rate of 9600 bps.

The "Mercury 2.0" robotic arm is a stand-alone electromechanical manipulator system developed by our team, capable of replicating most movements of a physical human arm. The current version of the robot is capable of movement along 8 DoFs [32]. Eight motors are attached in total on 


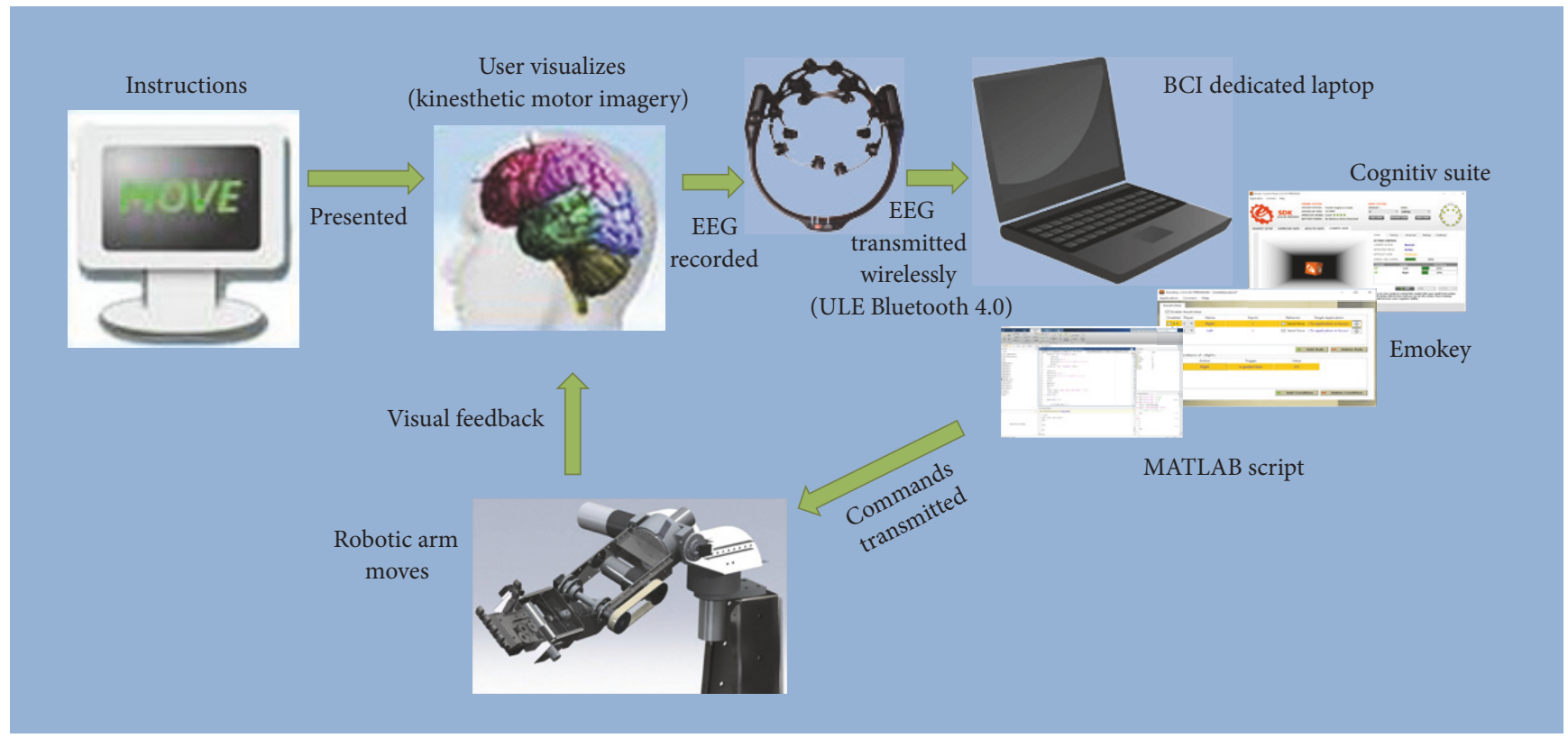

FIGURE 1: Brain-robot interface loop: using a wireless commercial EEG device for unobtrusiveness and simplicity of the system [12].

each arm, six of them being DC electric motors: two on the robotic shoulder joint, responsible for horizontal (1) and for vertical movement (2); one on the elbow joint, for horizontal movement (3); one on the wrist joint, for horizontal movement (4), one for rotation between the shoulder and elbow (5); and one for rotation between the elbow and wrist (6); finally, two servo motors add the ability of curling movement of the thumb and palm fingers (7) and (8). These 8 DoFs allow for responsive, fluid movement of the robotic arm and provide it with the ability to grab and manipulate small and light objects. Each arm functions with a single attached microcontroller, responsible for motors, connected to the dedicated computer system through a serial port at a Baud Rate of 9600 . The microcontroller translates the commands the computer system transmits into positional coordinates for each motor. System responsiveness has been measured to be approximately 0.2 seconds.

\subsection{Experimental Setup}

2.2.1. Ethical Approval and Recruitment. The institutional bioethics committee approved the experimental protocol [12, 31] and all subjects filled and signed an informed consent form prior to their participation. Criteria for participation included clinical diagnosis and radiological documentation of SCI (evaluated by ASIA Impairment Scale (AIS) [33] and/or neurological examination reporting the condition) or healthy participants [31]. Exclusion criteria were other neurological injury or disease (traumatic brain injury, central nervous system tumors, epilepsy, etc.), recent participation in an interventional study, other grave medical condition that could affect participation or the safety, hearing and visual impairments, illegal drug use, and chronic alcoholism.
TABLE 1: Descriptive statistics of age (mean and standard deviation) for both groups (SCI group and healthy controls).

\begin{tabular}{lc}
\hline Group & $\begin{array}{c}\text { Age } \\
\text { Mean (std) }\end{array}$ \\
\hline SCI & $46.0(17.64)$ \\
Healthy & $46.2(18.27)$ \\
\hline
\end{tabular}

Ten subjects with SCI ( 8 male, 2 female) and ten healthy controls (with an effort for being gender and age matched to the SCI group) were recruited in total. The SCI group had a mean age of 46.0 years (range 28-74, standard deviation (std) 17.64), while the healthy group had a mean age of 46.20 years (range 27-74, std 18.27), as shown in Table 1. The subjects had no prior experience in $\mathrm{BCI}$ or robotics.

2.2.2. Subject Assessment. For both groups demographics and medical history were collected including smoking habits, age, height, weight, Body Mass Index (BMI), and education level. BMI was calculated and the following classification was used: underweight $<18.5$, normal weight $=18.5-24.9$, overweight $=25-29.9$, and obesity $\geq 30$. For the SCI group, history data was also collected including age at injury, weight at injury, and cause of injury. Neurological examination was performed by a specialist physician using the International Standards for Neurological Classification of Spinal Cord Injury: severity of injury (classification in AIS), Neurological Level of Injury (NLI), motor scores for upper extremities (UEMS), lower extremities (LEMS), and total (Moto-Total), and sensory scores for light touch (LT), pin prick (PP), and total (SensoryTotal) were recorded [33]. Spasticity, if present, was also recorded using Ashworth [34] and Penn Spasm Frequency 
TABLE 2: Subjects by group (SCI and healthy controls): basic demographic data for both groups and cause of injury, ASIA Impairment Scale (AIS), and Neurological Level of Injury.

\begin{tabular}{lcccccccc}
\hline SCI group & Age & Gender & Cause & AIS & NLI & Healthy group & Age & Gender \\
\hline CSI-02-001 & 28 & $\mathrm{f}$ & MVA & ASIA C & C4 & CSI-04-001 & 27 & $\mathrm{f}$ \\
CSI-02-002 & 52 & $\mathrm{~m}$ & MVA & ASIA D & C4 & CSI-04-007 & 51 & $\mathrm{~m}$ \\
CSI-02-003 & 42 & $\mathrm{~m}$ & MVA & ASIA D & C8 & CSI-04-003 & 43 & $\mathrm{~m}$ \\
CSI-02-004 & 70 & $\mathrm{~m}$ & Fall & ASIA D & C5 & CSI-04-006 & 71 & $\mathrm{~m}$ \\
CSI-02-005 & 60 & $\mathrm{~m}$ & Fall & ASIA E & C6 & CSI-04-009 & 63 & $\mathrm{~m}$ \\
CSI-02-006 & 28 & $\mathrm{~m}$ & MVA & ASIA D & C5 & CSI-04-004 & 28 & $\mathrm{~m}$ \\
CSI-02-007 & 30 & $\mathrm{~m}$ & MVA & ASIA E & C5 & CSI-04-005 & 31 & $\mathrm{~m}$ \\
CSI-03-001 & 47 & $\mathrm{~m}$ & Fall & ASIA A & T7 & CSI-04-008 & 47 & $\mathrm{~m}$ \\
CSI-03-002 & 29 & $\mathrm{f}$ & MVA & ASIA B & T4 & CSI-04-002 & 27 & $\mathrm{f}$ \\
CSI-03-003 & 74 & $\mathrm{~m}$ & Other & ASIA B & T4 & CSI-04-010 & 74 & $\mathrm{~m}$ \\
\hline Mean & 46.00 & - & - & - & - & Mean & 46.20 & - \\
Std & 17.64 & - & - & - & - & Std & 18.27 & - \\
\hline
\end{tabular}

TABLE 3: Neurological evaluation scores of the SCI group.

\begin{tabular}{|c|c|c|c|c|c|c|}
\hline SCI group & Motor-Total & UEMS & LEMS & Sensory-Total & LT & $\mathrm{PP}$ \\
\hline CSI-02-001 & 48 & 24 & 24 & 224 & 112 & 112 \\
\hline CSI-02-002 & 84 & 39 & 45 & 183 & 94 & 89 \\
\hline CSI-02-003 & 98 & 48 & 50 & 224 & 112 & 112 \\
\hline CSI-02-004 & 98 & 48 & 50 & 223 & 111 & 112 \\
\hline CSI-02-005 & 100 & 50 & 50 & 224 & 112 & 112 \\
\hline CSI-02-006 & 100 & 50 & 50 & 222 & 111 & 111 \\
\hline CSI-02-007 & 100 & 50 & 50 & 224 & 112 & 112 \\
\hline CSI-03-001 & 56 & 50 & 6 & 121 & 58 & 63 \\
\hline CSI-03-002 & 50 & 50 & 0 & 156 & 78 & 78 \\
\hline CSI-03-003 & 54 & 50 & 4 & 156 & 78 & 78 \\
\hline Median & 91.00 & 50.00 & 47.50 & 222.50 & 111.0 & 111.50 \\
\hline Interquartile range & 47.00 & 4.30 & 44.50 & 68.00 & 34.00 & 34.00 \\
\hline
\end{tabular}

Scales [35]. Both groups were assessed for everyday life independence using the Spinal Cord Independence Measure (SCIM-III) [36], translated in Greek (g-SCIM-III) [37]. Total SCIM score and subscores for Self-Care (g-SCIM-III-SC), Respiration and Sphincter Control (g-SCIM-III-RS), and Mobility (g-SCIM-III-M) were recorded.

In the SCI group, 9 out of 10 patients had incomplete injury. Four patients were classified as AIS D (40\%), 2 were AIS B (20\%), 2 were AIS E (20\%), one had complete injury and was classified as AIS A (10\%), and one patient was classified as AIS C (10\%). Regarding level, 70\% were cervical injuries whereas the remaining 30\% were thoracic injuries. Moreover, the predominant cause of injury, in 60\% (6/10) of patients injury, was motor-vehicle accidents (MVA), while in $30 \%$ of participants the injury was induced by fall from heights (Fall) and one patient reported other causes. Table 2 depicts age and gender distributions for both groups and also cause of injury, AIS, and NLI by subject.

Based on neurological assessment, the $60 \%$ of patients that were classified as either AIS D or E showed approximately intact general motor function and excellent UEMS and LEMS (3/10 of patients scored 50 in both categories whereas $2 / 10$ scored 48 in UEMS and 50 in LEMS). The remaining $40 \%$ patients were classified as AIS A, B, or C and showed motor deficits, as presented in Table 3. The SCI group was therefore further grouped into positive outcome (60\%) and negative outcome (40\%) for further analysis as described in the statistical analysis section below. With regard to sensory skills, the patients with good outcome scored as high as the healthy controls in LT and PP sensory examination (Table 3).

Subject assessment also included subjective reporting of imagery capacity, using Vividness of Visual Imagery Questionnaire (VVIQ-II) [38] with eyes open that was assessed for total score and for each of the four scenarios (VVIQ1VVIQ4). Psychometric evaluation also entailed answering Beck Depression Inventory (BDI) [39] and Rosenberg SelfEsteem Questionnaire (RSEQ) [40], both translated in Greek $[41,42]$. After the participation in the experiment, both subject groups reported on HRI experience using the Godspeed robotics questionnaire [27], also translated in Greek [43]. Godspeed total score (GDSPD-Total) and subscores for Anthropomorphism (GDSPD-Anthr), Animacy (GDSPDAnim), Likeability (GDSPD-Like), Perceived Intelligence (GDSPD-Int), and Perceived Safety (GDSPD-Safe) were recorded. 


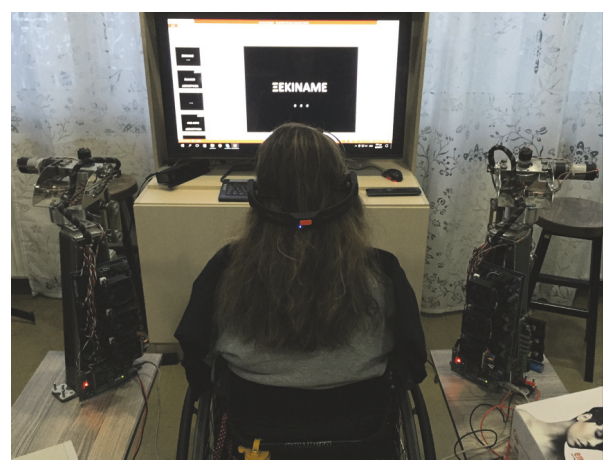

(a)

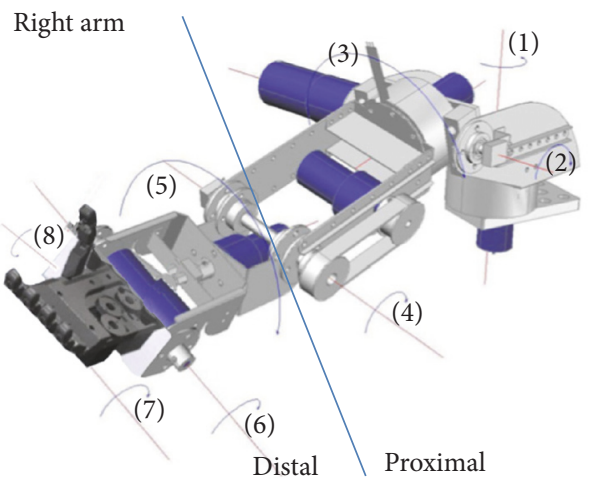

(c)

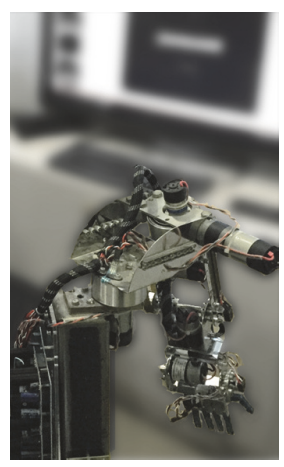

(b)

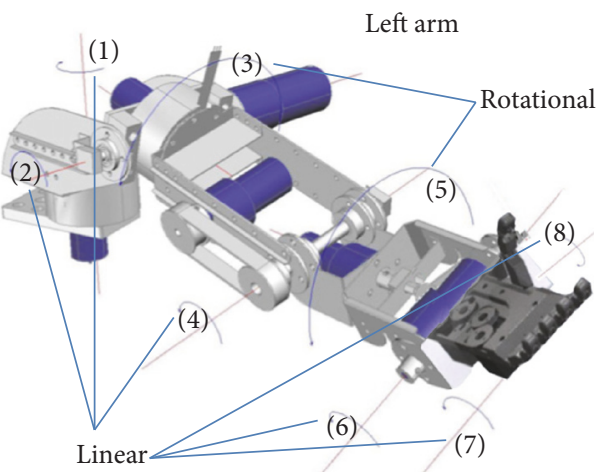

(d)

FIgURE 2: The experimental setup in Thess-AHAL. (a) SCI subject seated across the TV/computer monitor and between two robotic arms, wearing a wireless commercial EEG device; (b) close-up to a "Mercury 2.0" house-built robotic arm; (c) the right robotic arm, showing 8 possible DoFs, grouped into proximal and distal movements; (d) the left robotic arm, showing DoFs that result in rotational or linear movement. Each DoF allows movement towards two possible directions [12].

2.2.3. Experimental Procedure. As the experimental procedures have been also described in detail [12, 29], we will provide an overview hereby. The subjects were asked to perform Visual Motor Imagery (VMI), meaning they had to mentally rehearse 32 different movements of the upper extremities, while watching a set of arms performing randomly those movements on a computer screen. Each movement was shown 9 times in total. Randomly, walking and an oddball video were also shown. This VMI experience was aimed at preparing participants for the $\mathrm{BCI}$ classes they would have to later perform. This VMI procedure was also performed under high-resolution EEG recording, which will not be further elaborated hereby. The subjects had their arms, torso, and legs covered with a black curtain during VMI experience as well as during subsequent $\mathrm{BCI}$ training and $\mathrm{BCI}$ control to facilitate registration of the projected arms (in the case of VMI) or the robotic arms (in the case of BCI) into the body schema [28]. Also, in the case of VMI the projected arms were gendermatched to the subject.

Following VMI experience, the subjects sat across a computer monitor, between two Mercury 2.0 robotic arms, located in the Thessaloniki Active and Healthy Ageing Living Lab technology showcase room (Thess-AHALL, member of ENoLL) [44-46]. Subjects wore an Emotiv EPOC headset and they began training of the BCI modality using kinesthetic motor imagery (KMI) of movements of the left and the right arms. Action power threshold was set at 50\% and all subjects were above threshold during training [29]. Three imagery classes were trained (resting state, left, and right). Five training cycles were allowed for left and right, using continuous mental rehearsal of all movements of left and right arm, respectively (as in the videos that subjects watched) [47]. Training skills (Train-L) (Train-R) classes were recorded. Following BCI training, the subjects were given written commands in the monitor to move a specific DoF towards a specific direction. 32 different commands were given in total and the subjects were asked to use the trained KMI skill in order to control the robotic arms to perform those actions. Each command was projected for $30 \mathrm{sec}$ with a $5 \mathrm{sec}$ rest between them (Figure 2). During the $30 \mathrm{sec}$ period the subject attempted to perform the movement as many times as possible by giving the desired direction of imagery class (left or right). The BCI detected as correct (desired) any activation of the class corresponding to the arm currently targeted and gave the output to move the arm as per the instruction (positive feedback). Incorrect (undesired) activation that was detected resulted in an opposite motion of the target robotic arm (negative feedback). Desired and undesired commands that were detected by the BCI program were recorded through the MATLAB script. 
TABLE 4: House-developed performance rating scale for brain-robot interface control, tailored to the needs of the use case of the current experiment.

\begin{tabular}{lcc}
\hline \multicolumn{2}{c}{ Performance rating of brain-robot interface control based on ratio of desired to undesired detected \& classified mental commands } \\
Score & Rating & Description of rating \\
\hline 5 & Excellent & Arms were moving towards desired direction for most of the time \\
4 & Very Good & $5+$ commands were detected, minimal undesired commands \\
3 & Good & $4+$ commands were detected, only $2-3$ undesired commands \\
2 & Average & $3+$ commands were detected, but also up to +3 undesired commands \\
1 & Minimal & $1-2$ commands were detected or $3-4$ including undesired commands \\
0 & No control & or many commands were detected but most were undesired \\
\hline
\end{tabular}

Performance was rated in each different movement with a score from 0 to 5 , based on the ratio of desired mental commands to undesired mental commands that were detected and classified (Table 4) [12]. A total score for BCI performance (BCI-total) was then calculated for each subject, adding the scores for each movement ( $\max 160$ ) and the performance of each subject was also converted to a percentage (BCI\%). Subscores were calculated for the 16 movements of the left arm (BCI-L), the 16 movements of the right arm (BCI-R), the 16 movements of wrists, fingers, and thumbs (BCI-Distal), and the 16 movements of shoulder and elbow (BCI-Proximal), with max scores of 80 . Subscores were also calculated for 24 linear movements and 8 rotational movements but, to allow comparison, an average per movement score was calculated for both categories (BCI$\mathrm{Li} / 24$ and BCI-Ro/8).

\subsection{Statistical Analysis}

2.3.1. Statistical Tests. Statistical analysis was performed in IBM SPSS Statistics (version 23) and we set a significance level of 0.05 for all statistical tests. The variables were explored for normality assumption using two grouping schemes: (1) SCI and healthy groups as grouping factor for all subjects and (2) positive and negative outcome as grouping factor for SCI subjects.

Normality was explored using visual inspection of histograms, normal Q-Q plots and boxplots, skewness, and kurtosis [48-50] as well as using the normality tests (ShapiroWilk test and Kolmogorov-Smirnov Test) $[51,52]$. The variable age, in particular, was normally distributed for both groups (skewness: $0.407(\mathrm{SE}=0.687)$, kurtosis: $-1.418(\mathrm{SE}=1.334)$ for healthy group; skewness: $0.651(\mathrm{SE}=0.687)$, kurtosis: -0.752 $(\mathrm{SE}=1.334)$ for patient group).

Normality assumption was not met (1) for motor and sensory scores of neurological examination as well as SCIM scores, (2) for VVIQ1 and VVIQ3 scenarios and BDI in SCI and healthy groups, (3) for GDSPD-Safe in either grouping scheme, and (4) for training scores in outcome grouping. All other distributions met normality assumption. Depending on normality assumption group differences were explored using either Independent Samples t-test or Mann-Whitney $(U)$ test. Possible associations between quantitative variables were explored via Pearson correlation coefficient or Spearman's coefficient depending on normality. Other specific statistical tests or other specific groupings were used as described below. Please also refer to Supplementary Materials for a more detailed report of statistical analysis (available here).

2.3.2. Demographics, Somatometric Data, and Clinical Evaluation. Initially, we planned comparisons of demographic and somatometric data between SCI and healthy groups. Comparisons regarding the education level (basic studies, pregraduate level, graduate level, postgraduate level, and Ph.D. holders) were performed by Mann-Whitney (U) test, since our data are ordinal (Likert-type) [53]. Medical history and neurological data were analyzed via descriptive statistical methods. Moreover, we explored whether the smoking status is independent of the group or not using chi-square test.

2.3.3. Assessment Questionnaires and User Perception. Imagery capacity (VVIQ) and psychometric questionnaires (BDI and RSEQ) were analyzed between SCI and healthy groups, as well as between SCI subgroups of positive and negative outcomes. The scores of Godspeed and its subcategories (GDSPD-Total, GDSPD-Anthr, GDSPD-Anim, GDSPD-Like, GDSPD-Int, and GDSPD-Safe) were analyzed between patient and healthy groups, as well as between patient outcome groups. Finally, possible correlations were explored between Godspeed scores and BCI performance as well as Godspeed scores and VVIQ scores.

2.3.4. BCI Performance. BCI-total, BCI-L, BCI-R, and training skills (Train-L and Train-R) were analyzed between SCI and healthy groups as well as in SCI outcome subgroups. BCIDistal, BCI-Proximal, BCI-Li, and BCI-Ro were analyzed using descriptive statistics. We further explored differences in BCI performance and training scores between different groups of neurological levels of injury (cervical, thoracic) after testing for normality. Additionally, linear regression analysis was used to model the possible relationship between the independent variable BCI scores and the explanatory variable NLI using linear regression analysis. Possible correlations were further explored across groups (SCI/healthy) between BCI performance and (1) age, (2) imagery capacity (VVIQ), and (3) psychometric questionnaires (BDI, RSEQ) for both groups. 


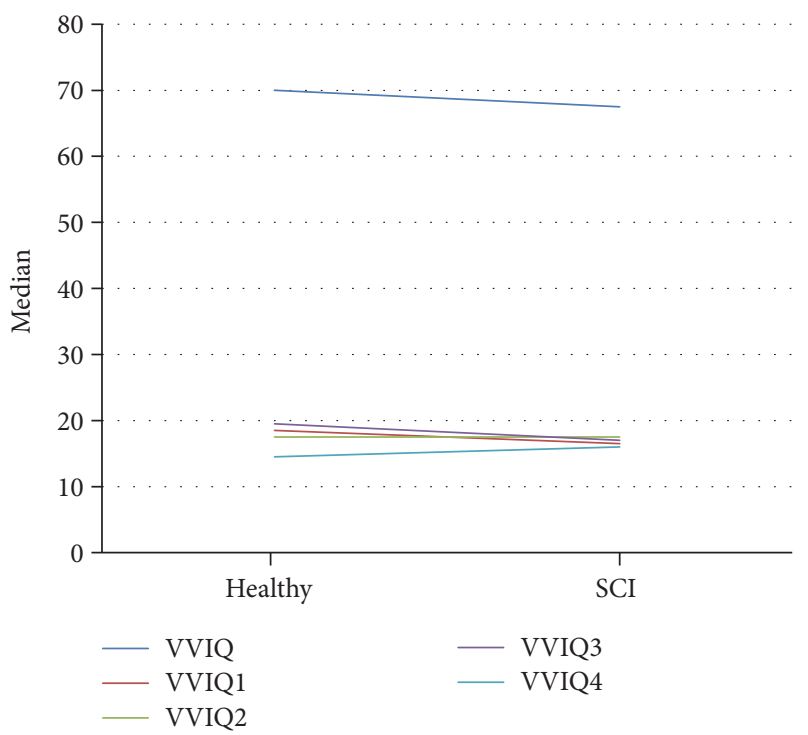

FIGURE 3: Scores of SCI and healthy groups at VVIQ test and its scenarios show no significant difference between groups.

\section{Results}

3.1. Demographics, Somatometric Data, and Clinical Evaluation. Planned comparisons of age between groups did not reveal any significant difference (Table 1). Regarding education level, no statistically significant difference between groups was shown. Group differences were not revealed in somatometric data either. Most of the injuries (4/10) happened when the participants were between 26.0 and 33.0 years of age. The second more vulnerable age range to injuries (MVAs) seems to be the age range of 50.0-57.0 years. Exploring whether the smoking status is independent of the group or not, we observed a significant association $\left(\chi^{2}=5.051, \mathrm{df}=\right.$ $1, \mathbf{p}=\mathbf{0 . 0 2 5}$ ), since $70 \%$ of the healthy group and only $20 \%$ of the SCI group were smokers. Subjects with positive outcome scored as high as the healthy subjects in g-SCIM-III test and its subcategories. In the remaining group, deviation in the performance at the g-SCIM-III test was found. Table 5 shows aggregated the most important statistical tests we performed and their results on demographics, clinical evaluation, and assessment questionnaires.

3.2. Assessment Questionnaires. VVIQ did not show any significant difference between SCI and healthy groups in total score or any of the scenarios. SCI group scored lower than healthy (Figure 3) but differences did not reach statistical significance. Regarding SCI outcome subgroups, no considerable group differences were revealed in VVIQ scores. Also, even though the SCI group showed increased depressive scores and decreased self-esteem compared to healthy group, group differences were not statistically significant (Figure 4).

3.3. BCI Performance. Planned between-group (SCI/healthy) comparisons of BCI performance revealed a statistically significant difference only in the control of right robotic arm (BCI-R: $t=2.592, \mathrm{df}=18, \mathbf{p}=\mathbf{0 . 0 1 8}$ ). Although BCI scores of SCI group were generally lower than those of control, group differences did not reach statistical significance. Also, regarding performance in different types of robotic arm movements (Table 6), based on the planned analysis, no significant outcomes were shown. Comparisons of BCI scores between SCI outcome subgroups (positive, negative) did not reveal any statistically significant difference (Figure 5). However, subgroup with positive outcome seems to score higher in total BCI control (Figure 6).

The ability of SCI subjects to control robotic arms did not differentiate depending on the injury location (cervical, thoracic). However, subjects with cervical injuries scored higher than those with thoracic injuries in BCI control. BCITotal was marginally negatively correlated to NLI in the SCI group ( $\mathbf{p}=\mathbf{0 . 0 6 4}$ ) (Figure 7 ). Negative correlations, but not statistically significant, were found between the BCI performance and the age (Table 6).

The training skill was not statistically different between SCI and healthy groups neither for the right hand, nor for the left hand, even if SCI subjects showed slightly lower training scores in the right hand and higher in the left hand than healthy participants. Comparing training scores between SCI subjects grouped by outcome (Figure 8), a marginally considerable difference was found only in training scores of left hand $(U=3.5, \mathbf{p}=\mathbf{0 . 0 6 8})$. SCI subjects with negative outcomes trained more efficiently in left hand compared to the subjects with positive outcome (Table 6). SCI subjects did not considerably differentiate their training scores depending on the level of injury. However, it was noted that Train-L was greater in thoracic SCI subgroup than those with cervical injuries whereas the opposite was the case for Train-R.

Total BCI scores were significantly negatively associated with VVIQ total scores $(r=-0.727, \mathbf{p}=\mathbf{0 . 0 1 7})$ and VVIQ3 $\left(r_{s}=-0.948, \mathbf{p}<\mathbf{0 . 0 0 1}\right)$ as well as the BCI-L scores and VVIQ3 $\left(r_{s}=-0.665, \mathbf{p}=\mathbf{0 . 0 3 6}\right)$ only in SCI group. However, other subcategories of VVIQ such as VVIQ1, VVIQ2, and VVIQ4 were not correlated to all BCI scores in SCI group (BCI-Total-VVIQ1: $r_{s}=-0.489, p=0.151 ; B C I-L-V V I Q 1:$ $r_{s}=-0.509, p=0.133 ; B C I-R-V V I Q 1: r_{s}=-0.194, p=$ 0.590; BCI-Total-VVIQ2: $r=-0.077, p=0.832$; BCI-LVVIQ2: $r=-0.073$, $p=0.840$; BCI-R-VVIQ2: $r=0.005$. $p=0.989 ;$ BCI-Total-VVIQ4: $r=-0.312, p=0.380 ; B C I-L-$ VVIQ4: $r=0.026, p=0.943$; BCI-R-VVIQ4: $r=-0.332, p=$ 0.348). With regard to BCI performance depending on the depressive symptomatology as assessed by BDI, significant negative correlation was found only between total BCI scores and BDI scores in healthy participants $\left(r_{s}=-0.719, \mathbf{p}=\right.$ 0.019). Correlations explored between all BCI scores and scores at Rosenberg Self-Esteem Scale did not reach statistical significance for both groups (Healthy-BCI-Total-Rosenberg: $r=0.150, p=0.679$; BCI-L-Rosenberg: $r=0.054 . p=0.882$; BCI-R-Rosenberg: $r=0.115, p=0.751$; SCI: BCI-TotalRosenberg: $r=-0.067, p=0.854$; BCI-L-Rosenberg: $r=$ -0.397, $p=0.255$; BCI-R-Rosenberg: $r=0.369, p=0.294)$.

3.4. User Perception. Based on planned analysis of HRI characteristics of the robotic arms, SCI and healthy groups did not present significant differences in their answers in any subcategory (GDSPD-Anthr: $t=1.504$, $\mathrm{df}=18, p=0.150$; 


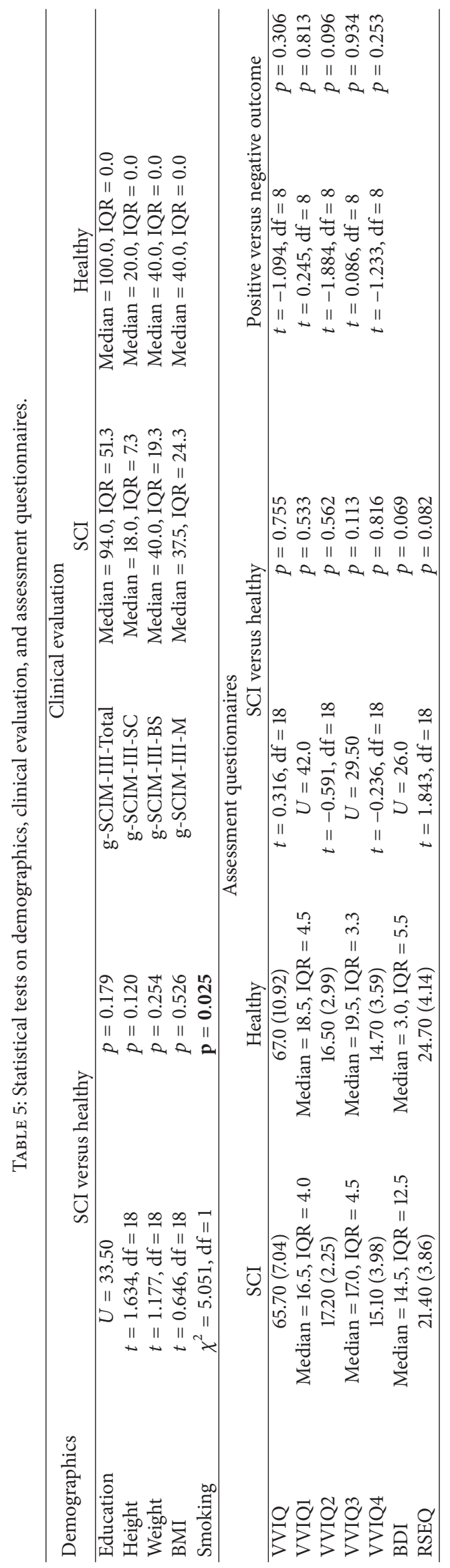




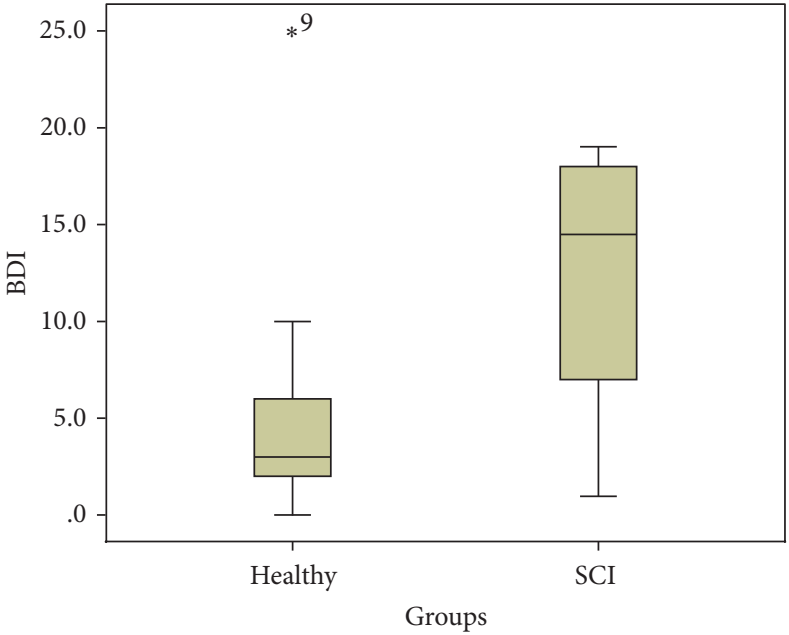

(a)

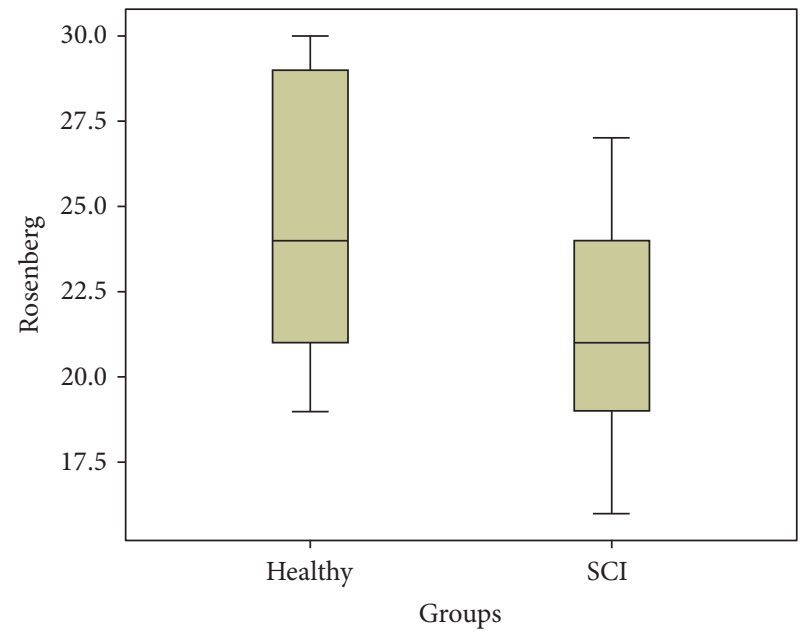

(b)

Figure 4: SCI scores at Beck Depression Inventory are increased while at Rosenberg Self-Esteem Scale they are decreased, compared to healthy controls, but findings are not statistically significant. ${ }^{*}$ Extreme value.

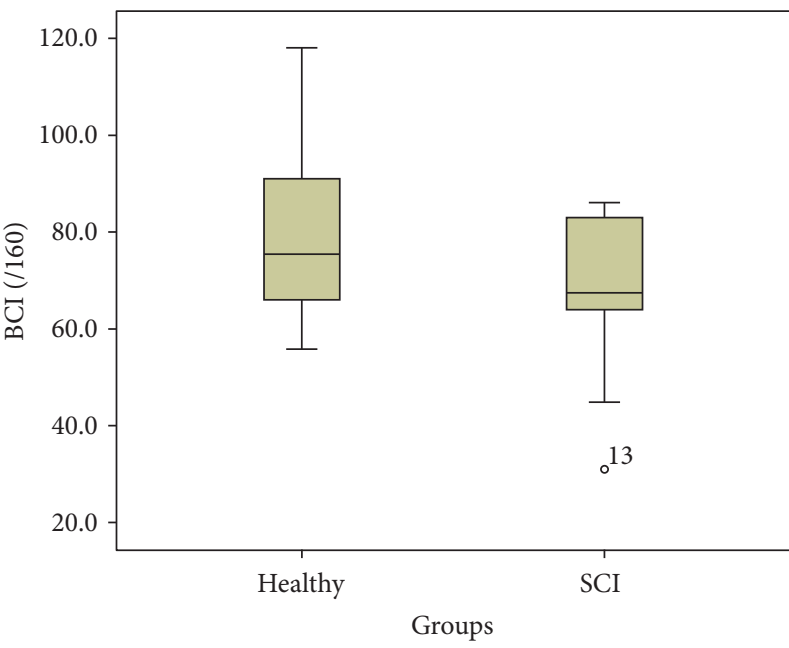

(a)

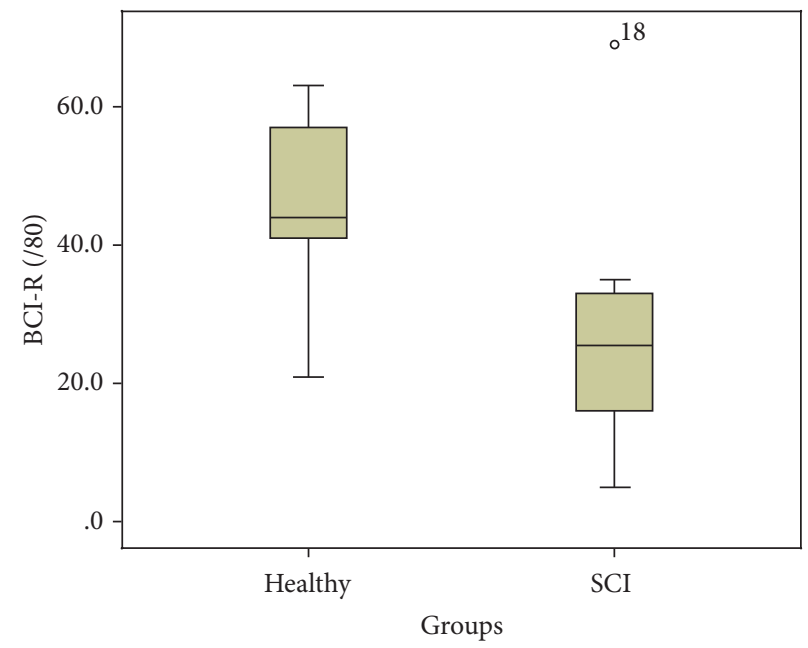

(b)

FIGURE 5: BCI performance (BCI-Total) in the control of robotic arms and in the control of the right robotic arm (BCI-R) was lower for SCI group, but not statistically significant. o: outlier.

GDSPD-Anim: $t=0.611, \mathrm{df}=18, p=0.549 ;$ GDSPD-Like: $t=-0.217, \mathrm{df}=18, p=0.831 ; G D S P D-I n t: t=-0.047, \mathrm{df}=18$, $p=0.963$; GDSPD-Safe: $U=42.0, p=0.536$ ) of Godspeed questionnaire. Additionally, there was no difference between groups in GDSPD-Total $(t=0.427, \mathrm{df}=18, p=0.675)$.

The scores are illustrated in Figure 9 (GDSPD (/120) (mean (SD)), healthy: 80.80 (14.78); SCI: 78.00 (14.57); GDSPD-Anthr, healthy: 13.60 (4.25); SCI: 10.80 (4.08); GDSPD-Anim, healthy: 18.90 (5.11); SCI: 17.70 (3.53); GDSPD-Like, healthy: 18.50 (3.87); SCI: 18.90 (4.36); GDSPD-Int, healthy: 17.70 (4.60); SCI: 17.80 (4.92); GDSPDSafe, healthy $($ median $=12.0, \mathrm{IQR}=5.3)$; SCI $($ median $=13.50$, IQR = 4.5)).
Godspeed's scores were further explored after grouping by outcome (positive, negative). Marginally significant difference was found only in Anthropomorphism $(t=2.251, \mathrm{df}=$ $8, p=0.054)(G D S P D$ (/120): $t=1.918, \mathrm{df}=8,0.091$; GDSPSAnim: $t=1.382, \mathrm{df}=8, p=0.204 ;$ GDSPD-Like: $t=1.289$, df $=8, p=0.233$; GDSPD-Int: $t=1.343, \mathrm{df}=8, p=0.216$; GDSPD-Safe: $U=10.50, p=0.741)$. More precisely, SCI subjects with negative outcome scored higher than those with positive outcome in this Godspeed's subcategory (negative outcome: 13.75 (3.30); positive outcome: 8.83 (3.43)).

Significant correlation between Godspeed's and BCI scores was not revealed for any group. GDSPD-Anthr and GDSPD-Int were positively correlated to VVIQ4 and VVIQ1 


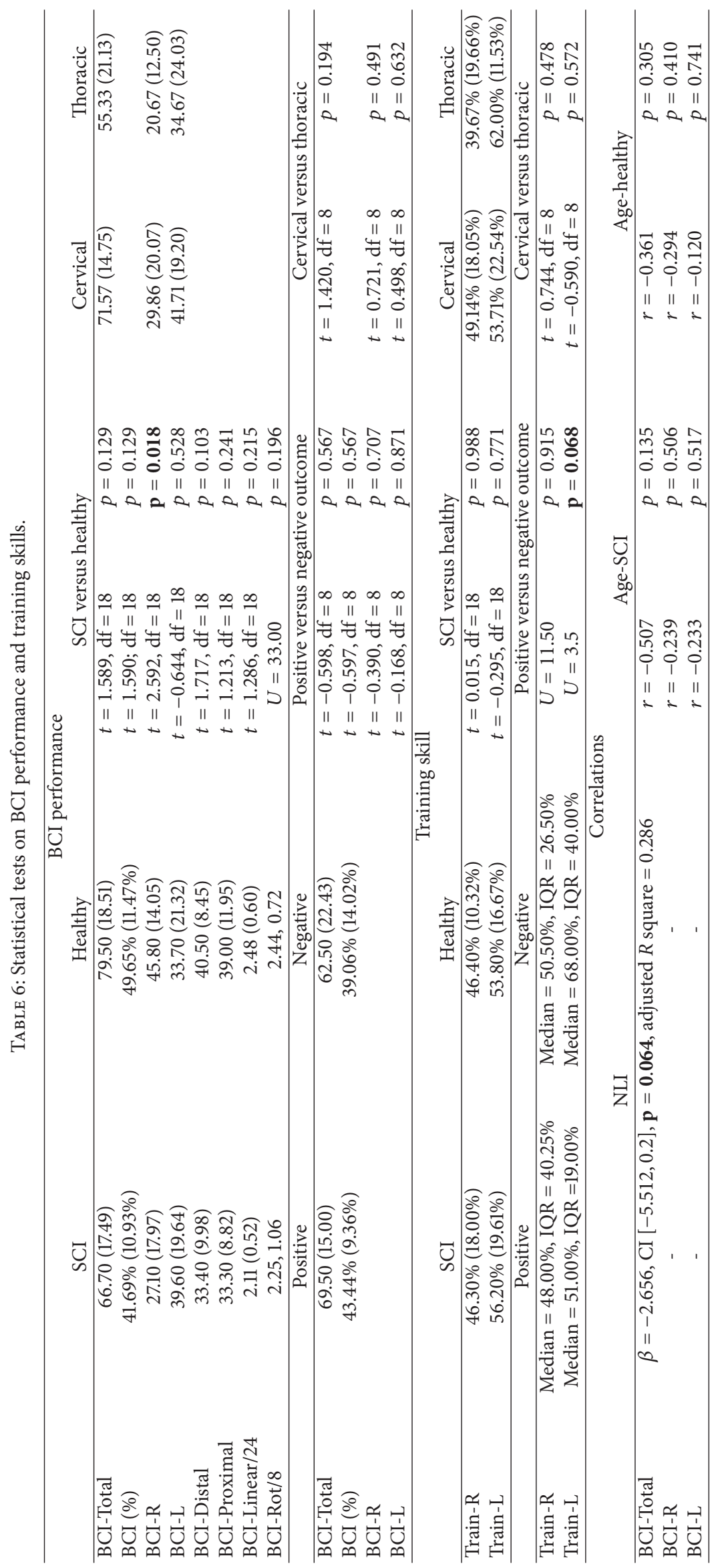




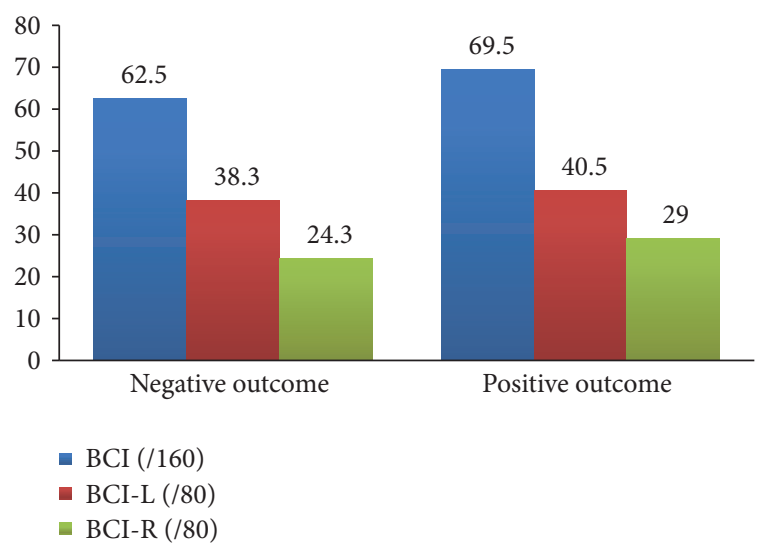

FIGURE 6: BCI scores of SCI subjects grouped by neurological outcome. Subjects with positive outcome performed nonsignificantly better in BCI control than those with negative.

scenario's scores, respectively, only in healthy (GDSPD-Int, VVIQ4: $r=0.654, p=0.040$; GDSPD-Anthr, VVIQ1: $r=$ $0.629, p=\mathbf{0 . 0 5 2})$. In SCI group, GDSPD-Safe was positively associated with scores in VVIQ total $(r=0.696, p=\mathbf{0 . 0 2 5})$ and VVIQ1 $(r=0.780, p=\mathbf{0 . 0 0 8})$.

\section{Discussion}

4.1. User Perception and Performance Assessment. Our results show that healthy controls performed better (49.65\%) than the SCI group (41.65\%) in BCI control and so did subjects with positive neurological outcome (43.44\%) when compared to subjects with negative neurological outcome (39.6\%). Both those observations did not reach statistical significance but their interpretation is not as straightforward. Since no patients with complete tetraplegia were included, these findings probably cannot be attributed to some type of "thought extinction process" [54]. Indeed, self-reported imagery capacity (as measured by VVIQ) was found to be nonsignificantly lower in SCI group than in healthy subjects but, on the other hand, subjects with cervical injuries fared better than those subjects with thoracic injuries. Thus the group with injury that could affect upper extremity neural circuits outperformed those subjects that did not have direct injury to those circuits. Moreover this finding was further reinforced by a marginally significant (negative) correlation of BCI performance to neurological level of injury, meaning that the higher the level of injury, the better the subjects performed.

Possible explanations for performance differences could be explored along the lines of depression [55] and motivation $[56,57]$. Indeed the SCI group showed increased depressive scores (measured by BDI) and lower self-esteem scores (measured by RSEQ), despite both findings not being statistically significant. Our research protocol did not include any official questionnaire to assess SCI and healthy subject with regard to motivation. Only by anecdotal evidence, during informal debriefing discussions that we held with participants, subjects with thoracic injuries tended to consider somewhat irrelevant this experimental setup to their condition. While reciprocal sensorimotor pathways and sensorimotor cortex representations are affected regardless of the level of SCI, those subjects felt that their clinical condition and independence demands were not addressed by robotic arms technology.

The HRI characteristics of the robot were measured (after the participation) by Godspeed robotics questionnaire. It seems that user perception did not differ significantly between SCI and healthy subjects. While in Figure 9 we show absolute values for the total questionnaire score and its subscores, since no standard for direct comparison of those can be identified, we feel that intergroup comparisons are more useful. As such, we believe that this is a positive finding, interpreting it as users with SCI appreciating the robot more or less the same as their healthy counterparts. Among SCI subgroups, those with negative outcome gave higher scores to Anthropomorphism of the robot (mean 13.75 out of max 25) than those with positive outcome (mean 8.83 out of max 25), a finding that can be considered marginally significant (see Figure 9(b)). Indirectly, since user perception did not correlate with BCI performance and did not vary between SCI and healthy subjects in all other comparisons, perhaps this could be attributed to psychometric attributes of the participants and it should be further investigated in an SCI group with wider participation.

As we mentioned, no statistically significant negative correlations were found between BCI performance and the age of the subjects, a finding that also needs further study in order to be validated. As much of the BCI research field endeavors have been shaped around the issue of providing affordable, acceptable, and useful assistive systems for the benefit of the disabled, the particular characteristics of elderly patients should be the focus of investigation $[58,59]$.

Finally, BCI performance was significantly negatively associated $(p=0.017)$ with imagery capacity (as selfreported by VVIQ), a seemingly nonsensical finding. That could probably be attributed to one of the scenarios (VVIQ3 $p<0.001)$ skewing the total. We have insufficient data to interpret this, but until further evidence our hesitant (since this is an established tool [60]) suggestion would be to either to use a different research tool to measure imagery capacity or to omit the 3rd scenario of the VVIQ in the context of BCI controlled robotic arm experiments.

Some correlation was also revealed between reported imagery capacity and Godspeed questionnaire. SCI subjects that had higher scores in VVIQ rated Perceived Safety higher $(p=0.025)$. This finding was strongly influence by VVIQ 1st scenario $(p=0.008)$ since it was not present for the other scenarios. Said scenario asks of the subjects to think of some relative or friend whom they frequently see and consider their picture, a mental task that could induce a sense of familiarity or attachment and perhaps affect their answer on the Perceived Safety questions [61]. On the other hand, healthy subjects that had higher scores in VVIQ 1st and 4th scenarios rated Perceived Intelligence and Anthropomorphism higher too, findings that are not explained by the content of those scenarios. Perhaps their significance is marginal ( $p=0.052$ and $p=0.040$, resp.) but whether these trends persist or not with a wider user base is something that should be investigated before drawing an accurate conclusion. 


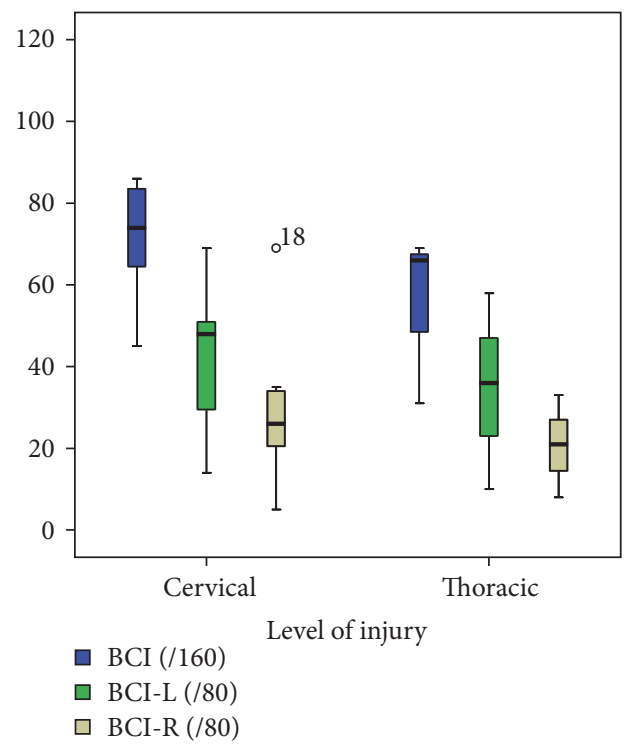

(a)

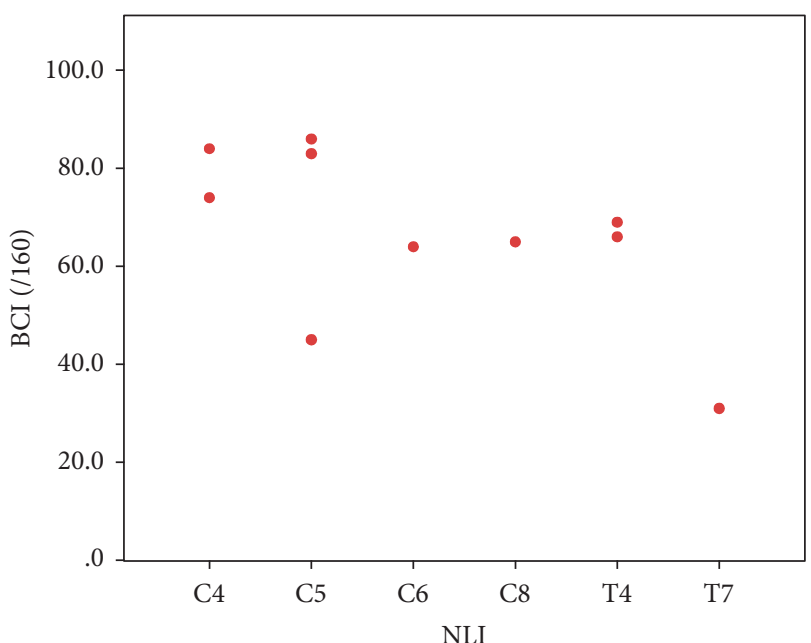

(b)

FIGURE 7: BCI performance of SCI group depending on the level of injury (a) and significantly (marginal inversely) correlated to NLI. $\circ$ : outlier.

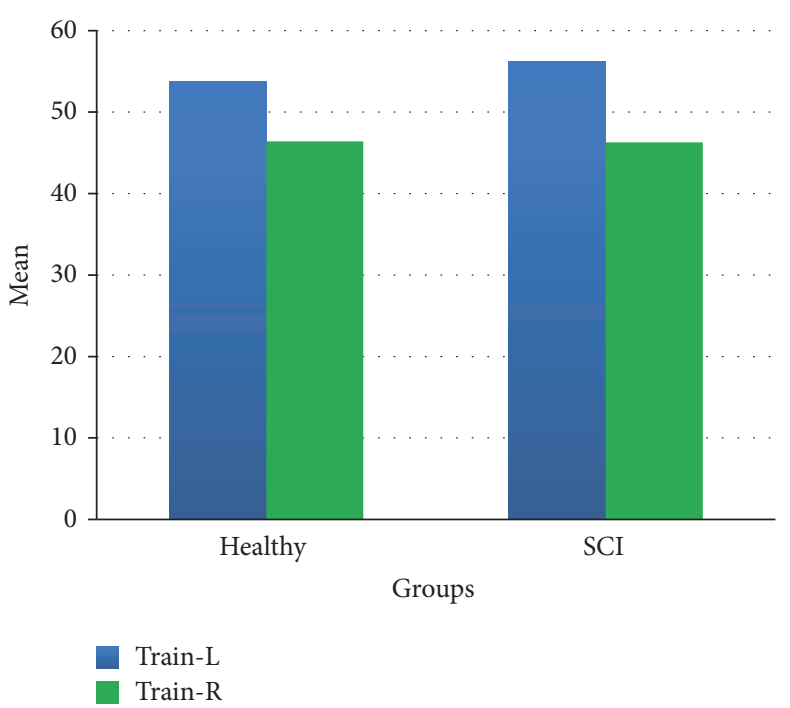

(a)

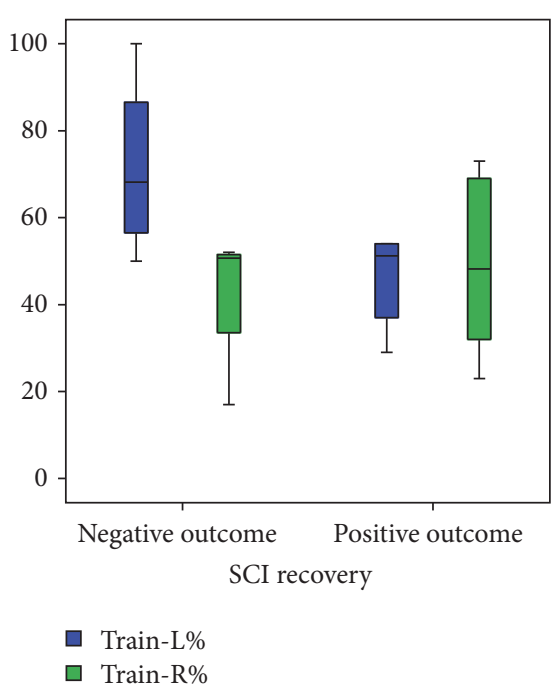

(b)

FIGURE 8: Train-L and Train-R, mean values_-grouped by SCI or healthy group (a) and median values_-grouped by outcome (b). SCI subjects with negative outcome performed marginally better than those with positive.

Our work adds onto an envisaged puzzle of BCI technology and uses case scenarios with an emphasis on affordability (off-the-shelf component system) and realism at a reasonable accuracy. Multi DoF robotics control by users with SCI has been proven to be feasible even by commercial-class wireless BCI. Nonetheless, repeated training in several BCI sessions is probably needed to improve performance by the end-users. Although users with complete lesions by definition would benefit more from ongoing research in robotics and BCIs, the use of brain controlled assistive technologies holds special importance for users with incomplete injuries too [62]. As a result of preserved reciprocal neural pathway communication, robotic-assisted training and brain controlled functional stimulation have been shown to improve arm motor function of those patients $[63,64]$ but expectations from assistive technologies and motivation to use them should be among key targets for further user experience investigations.

4.2. Further Technological Development. Our main development directions include (1) improving accuracy, speed, responsiveness, fluidity, and efficiency; (2) enhancing the integration of the system into the operator's perceived body 


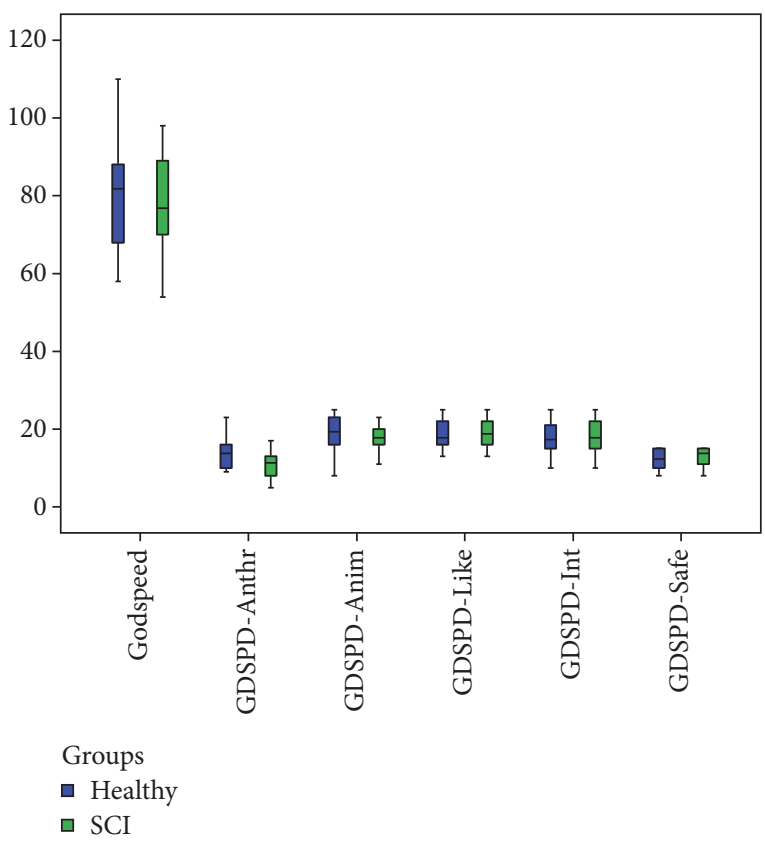

(a)

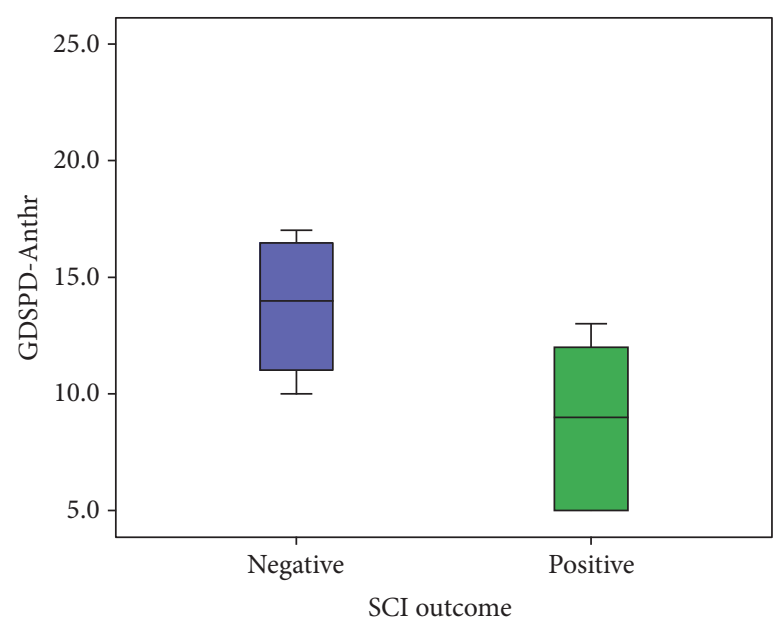

(b)

FIGURE 9: Answers provided to all Godspeed scores from SCI and healthy groups (a) showing no significant difference between groups. Anthropomorphism (b) was rated marginally higher by SCI subjects with negative outcome than those with positive.

mental image [17]. The pipeline that we overview below is tested offline, since several aspects, such as the solution of the inverse problem, entail high computational cost and are planned to be realized with dedicated processing units and use of artificial intelligence (AI) [12]. We are currently developing and testing a novel BCI decoding algorithm. Our goal is to increase discriminative ability to multiple MI classes projecting sensor data to cortical source domain $[65,66]$. The COLIN27 anatomy is used [67], but tests are also made on individual head models. Our approach is based on studies indicating that the features extracted from the source domain enhance the discrimination [68-70], since the higher dimensional space of sources incorporates anatomical and physiological information. Spatial features are extracted in predefined Regions of Interest (ROIs) [12] and Common Spatial Pattern (CSP) filters [71] are calculated on each ROI. Tuning and feature selection using a linear classifier (LDA) are a crucial part in order to determine the most valuable ROIs and improve BCI performance. The use of time-frequency and connectivity features in conjunction will also be explored.

4.3. Limitations. As we have already mentioned [12], multiple MI class discrimination is a challenge that state-of-the-art noninvasive BCI technology has yet to meet with success. Depending on a proprietary BCI algorithm, such as in our case, further underlines this problem, as it was not tailored to our specific needs. While the commercial-class device meets the use case needs and will be retained in future implementations, the proprietary $\mathrm{BCI}$ algorithm will be substituted with the one currently under development, in order to explore and reach practically usable performance of the entire system. This setup will be used in future experiments, including tests on the EEG data that have been already recorded. In general, off-the-shelf BCI technology seems to be able to meet the demands set by the field for unobtrusiveness, portability, and simplicity but it is possible that multi-DoF control may be not feasible or impractical without extensive use of supportive AI and advances in electronics charged with decision-making. Finally, from the investigation's perspective, although our study is one among those featuring adequate sized sample of disabled end-users for BCI technology [72, 73], our approach would benefit from wider subject participation for validation and extraction of statistically significant-and relevant to the advancement of the research field-results.

\section{Conclusions}

Much of the BCI research endeavor has been shaped around the issue of providing affordable, accurate, and real-time assistive systems for the benefit of the disabled. This piece of research adds onto that envisaged puzzle, but tackling affordability (off-the-shelf component system) of realistic scenarios at a reasonable accuracy. Healthy controls, SCI subjects with positive neurological outcome, and subjects with cervical injuries performed better in BCI control. Depression and motivation could play significant roles in BCI and robotics control. Reported imagery capacity was nonsignificantly lower for SCI subjects. User perception of the robot did not differ between SCI and healthy subjects, but, among SCI participants, those with negative outcome rated anthropomorphic characteristics higher. More BCI 
sessions are expected to improve performance of SCI and healthy subjects. The herein results demonstrate that by developing BCI decoding algorithms capable of true control of multiple DoFs and addressing the computational cost of online implementation of such an approach, it could be within short-term reach. While maintaining requirements for wireless, unobtrusive solutions constitutes challenges yet to be met, the exploitability of such a system by real patients at a reasonable accuracy cannot be understated. Improving electronics, fluidity, and accuracy of the robotic system, enhancing HRI experience, and implementing a sourcebased BCI algorithm for multiple class control, as well as further investigations with disabled users, are among our next steps.

\section{Ethical Approval}

This study was conducted in accordance with the Declaration of Helsinki (1964) and its following amendments. The Bioethics \& Ethics Committee of Faculty of Medicine, Aristotle University of Thessaloniki, approved the study.

\section{Consent}

All experiments were conducted with the subjects' understanding and written informed consent.

\section{Conflicts of Interest}

The authors declare that there are no conflicts of interest regarding the publication of this article.

\section{Acknowledgments}

This work was supported in part by 2013 Mario Boni Grant from Cervical Spine Research Society-European Section, awarded to CSI: Brainwave Project. This work was supported in part by the European Union's Horizon 2020 UNCAP project (Grant no. 643555).

\section{Supplementary Materials}

Report of statistical analysis. Table 1: descriptive statistics of age for both groups (healthy, SCI). Figure 1: the education level of the healthy and SCI participants. Figure 2: BMI categories across groups (the percentage of participants in each category is displayed on the bars). Figure 3: SCI subjects' age at injury. Table 2: SCI subjects' weight at injury. Figure 4: reported causes of injury. Figure 5: grouping the reported cause of injury by SCI subjects' age. Figure 6: SCI subjects' injury severity as assessed by ASIA Impairment Scale. Figure 7: neurological level of SCI subjects' injuries. Table 3: neurological evaluation scores of the SCI group. Figure 8: median scores of both groups at g-SCIM-III test and its subcategories. Figure 9: smoking status of both healthy and SCI group. Figure 10: performance of both groups at VVIQ test and its subcategories. Figure 11: scores at VVIQ questionnaire and its subcategories of both SCI outcome subgroups. Figure 12: scores of both groups at Beck Depression Inventory (on left hand) and Rosenberg Self-Esteem Scale. Figure 13: BCI performance of both groups in the control of right hand (on the left) and both hands (on the right). Figure 14: BCI scores in SCI group depending on their ASIA classification. Figure 15: BCI performance of SCI group depending on the level of injury. Figure 16: marginally negative correlation between total BCI scores and NLI. Figure 17: mean training scores of both groups at left and right hand. Figure 18: training scores of SCI groups depending on their ASIA classification. Figure 19: mean BCI and training performance depending on the level of injury. Figure 20: answers provided to Godspeed questionnaire from both groups. Figure 21: scores of SCI subjects at GDSPD-Anthr depending on outcome. Figure 22: BCI performance of both groups in different categories of movements. (Supplementary Materials)

\section{References}

[1] L. R. Hochberg, D. Bacher, B. Jarosiewicz et al., "Reach and grasp by people with tetraplegia using a neurally controlled robotic arm," Nature, vol. 485, no. 7398, pp. 372-375, 2012.

[2] J. L. Collinger, B. Wodlinger, J. E. Downey et al., "Highperformance neuroprosthetic control by an individual with tetraplegia," The Lancet, vol. 381, no. 9866, pp. 557-564, 2013.

[3] A. R. C. Donati, S. Shokur, E. Morya et al., "Long-term training with a brain-machine interface-based gait protocol induces partial neurological recovery in paraplegic patients," Scientific Reports, vol. 6, no. 1, p. 30383, 2016.

[4] M. Alam, W. Rodrigues, B. N. Pham, and N. V. Thakor, "Brainmachine interface facilitated neurorehabilitation via spinal stimulation after spinal cord injury: Recent progress and future perspectives," Brain Research, vol. 1646, pp. 25-33, 2016.

[5] R. R. Harrison, R. J. Kier, C. A. Chestek et al., "Wireless neural recording with single low-power integrated circuit," IEEE Transactions on Neural Systems and Rehabilitation Engineering, vol. 17, no. 4, pp. 322-329, 2009.

[6] A. V. Nurmikko, J. P. Donoghue, L. R. Hochberg et al., "Listening to brain microcircuits for interfacing with external worldprogress in wireless implantable microelectronic neuroengineering devices," Proceedings of the IEEE, vol. 98, no. 3, pp. 375388, 2010.

[7] J. L. Collinger, S. Foldes, T. M. Bruns, B. Wodlinger, R. Gaunt, and D. J. Weber, "Neuroprosthetic technology for individuals with spinal cord injury," The Journal of Spinal Cord Medicine, vol. 36, no. 4, pp. 258-272, 2013.

[8] P. H. Peckham and J. S. Knutson, "Functional electrical stimulation for neuromuscular applications," Annual Review of Biomedical Engineering, vol. 7, pp. 327-360, 2005.

[9] L. Pignolo, "Robotics in neuro-rehabilitation," Journal of Rehabilitation Medicine, vol. 41, no. 12, pp. 955-960, 2009.

[10] B. He, Ed., Modeling, Imaging of Bioelectrical Activity, Springer US, Boston, MA, USA, 2005.

[11] P. L. Nunez and R. Srinivasan, "Electric Fields of the Brain: The neurophysics of EEG," Electric Fields of the Brain: The neurophysics of EEG, pp. 1-611, 2009.

[12] A. Athanasiou, I. Xygonakis, N. Pandria et al., "Towards Rehabilitation Robotics: Off-the-Shelf BCI Control of Anthropomorphic Robotic Arms," BioMed Research International, vol. 2017, pp. 1-17, 2017. 
[13] S. Baillet, "Magnetoencephalography for brain electrophysiology and imaging," Nature Neuroscience, vol. 20, no. 3, pp. 327339, 2017.

[14] J. Meng, S. Zhang, A. Bekyo, J. Olsoe, B. Baxter, and B. He, "Noninvasive electroencephalogram based control of a robotic arm for reach and grasp tasks," Scientific Reports, vol. 6, no. 1, article 38565, 2016.

[15] Y. Kim and A. M. Cook, "Manipulation and Mobility Aids," in Electronic Devices for Rehabilitation, J. G. Webster, Ed., Wiley, London, 1985.

[16] M. Hillman, "2 Rehabilitation Robotics from Past to Present A Historical Perspective," in Advances in Rehabilitation Robotics, pp. 25-44, Springer Berlin Heidelberg, 2006.

[17] W. Seamone and G. Schmeisser, Evaluation of the APL/JHU Robot Arm Work Station, 1986.

[18] C. P. Mason and E. Peizer, "Medical Manipulator for Quadriplegics, Colloques IRIA," in Proceedings of the in International Conference on Telemanipulators for the Physically Handicapped, pp. 309-312, 1978.

[19] H. H. Kwee, M. Tramblay, R. Barbier et al., "First experimentation of the spartacus telethesis in a clinical environment," Paraplegia, vol. 21, no. 5, pp. 275-286, 1983.

[20] Y. Wakita, W.-K. Yoon, and N. Yamanobe, "User evaluation to apply the robotic arm RAPUDA for an upper-limb disabilities Patient's Daily Life," in Proceedings of the 2012 IEEE International Conference on Robotics and Biomimetics, ROBIO 2012, pp. 1482-1487, China, December 2012.

[21] V. Maheu, P. S. Archambault, J. Frappier, and F. Routhier, "Evaluation of the JACO robotic arm: Clinico-economic study for powered wheelchair users with upper-extremity disabilities," in Proceedings of the Rehab Week Zurich 2011 - 2011 IEEE International Conference on Rehabilitation Robotics, ICORR 2011, Switzerland, July 2011.

[22] M. S. Johannes, J. D. Bigelow, J. M. Burck, S. D. Harshbarger, M. V. Kozlowski, and T. Van Doren, "An overview of the developmental process for the modular prosthetic limb," Johns Hopkins APL Technical Digest, vol. 30, no. 3, pp. 207-216, 2011.

[23] J. J. Daly and J. R. Wolpaw, "Brain-computer interfaces in neurological rehabilitation," The Lancet Neurology, vol. 7, no. 11, pp. 1032-1043, 2008.

[24] M. Gomez-Rodriguez, M. Grosse-Wentrup, J. Hill, A. Gharabaghi, B. Scholkopf, and J. Peters, "Towards brain-robot interfaces in stroke rehabilitation," in Proceedings of the IEEE International Conference on Rehabilitative Robotics, vol. 2011, 2011.

[25] K. K. Ang, C. Guan, K. S. Chua et al., "A clinical study of motor imagery-based brain-computer interface for upper limb robotic rehabilitation," in Proceedings of the Annual International Conference of the IEEE Engineering in Medicine and Biology Society (EMBC '09), pp. 5981-5984, Minneapolis, Minn, USA, September 2009.

[26] K. Dautenhahn, "Methodology \& themes of human-robot interaction: a growing research field," International Journal of Advanced Robotic Systems, vol. 4, no. 1, pp. 103-108, 2007.

[27] C. Bartneck, D. Kulić, E. Croft, and S. Zoghbi, "Measurement instruments for the anthropomorphism, animacy, likeability, perceived intelligence, and perceived safety of robots," International Journal of Social Robotics, vol. 1, no. 1, pp. 71-81, 2009.

[28] O. Christ and M. Reiner, "Perspectives and possible applications of the rubber hand and virtual hand illusion in non-invasive rehabilitation: Technological improvements and their consequences," Neuroscience \& Biobehavioral Reviews, vol. 44, pp. 3344, 2014.

[29] G. Arfaras, A. Athanasiou, P. Niki et al., "Visual Versus Kinesthetic Motor Imagery for BCI Control of Robotic Arms (Mercury 2.0)," in Proceedings of the 2017 IEEE 30th International Symposium on Computer-Based Medical Systems (CBMS), pp. 440-445, Thessaloniki, June 2017.

[30] A. Athanasiou, G. Arfaras, I. Xygonakis et al., "Commercial BCI Control and Functional Brain Networks in Spinal Cord Injury: A Proof-of-Concept," in Proceedings of the 2017 IEEE 30th International Symposium on Computer-Based Medical Systems (CBMS), pp. 262-267, Thessaloniki, June 2017.

[31] "Brainwave control of a wearable robotic arm for rehabilitation and neurophysiological study in cervical spine injury (CSI:Brainwave)," https://clinicaltrials.gov/ct2/show/ NCT02443558.

[32] N. Moustakas, P. Kartsidis, A. Athanasiou, A. Astaras, and P. D. Bamidis, "Development of MERCURY version 2.0 robotic arms for rehabilitation applications," in Proceedings of the 8th ACM International Conference on PErvasive Technologies Related to Assistive Environments (PETRA '15), July 2015.

[33] S. C. Kirshblum, S. P. Burns, F. Biering-Sorensen et al., "International standards for neurological classification of spinal cord injury (revised 2011)," The Journal of Spinal Cord Medicine, vol. 34, no. 6, pp. 535-546, 2011.

[34] R. W. Bohannon and M. B. Smith, "Interrater reliability of a modified Ashworth scale of muscle spasticity," Physical Therapy in Sport, vol. 67, no. 2, pp. 206-207, 1987.

[35] J. T. C. Hsieh, D. L. Wolfe, W. C. Miller, and A. Curt, "Spasticity outcome measures in spinal cord injury: Psychometric properties and clinical utility," Spinal Cord, vol. 46, no. 2, pp. 86-95, 2008.

[36] M. Itzkovich, I. Gelernter, and F. Biering-Sorensen, "The Spinal Cord Independence Measure (SCIM) version III: Reliability and validity in a multi-center international study," Disability and Rehabilitation, vol. 29, no. 24, pp. 1926-1933, 2007.

[37] A. Athanasiou, A. Alexandrou, E. Paraskevopoulos, N. Foroglou, A. Prassas, and P. D. Bamidis, "Towards a Greek adaptation of the Spinal Cord Independence Measure (SCIM)," in in proceedings of the 15th European Congress of Neurosurgery (EANS 14), pp. 181-184, 2015.

[38] D. F. Marks, "Directions in Mental-Imagery Research," Journal of Mental Imagery, vol. 19, no. 3-4, pp. 153-167, 1995.

[39] A. T. Beck, R. A. Steer, and M. G. Garbin, "Psychometric properties of the Beck Depression Inventory: twenty-five years of evaluation," Clinical Psychology Review, vol. 8, no. 1, pp. 77100, 1988.

[40] W. Petersen, "Society and the Adolescent Self-Image. Morris Rosenberg. Princeton University Press, Princeton, N.J., 1965. xii + 326 pp. \$6.50," Science, vol. 148, no. 3671, p. 804, 1965.

[41] M. Giannakou, P. Roussi, M.-E. Kosmides, G. Kiosseoglou, A. Adamopoulou, and G. Garyfallos, "Adaptation of the beck depression inventory-II to greek population," Hellenic Journal of Psychology, vol. 10, no. 2, pp. 120-146, 2013.

[42] C. Galanou, M. Galanakis, E. Alexopoulos, and C. Darviri, "Rosenberg Self-Esteem Scale Greek Validation on Student Sample," Psychology, vol. 05, no. 08, pp. 819-827, 2014.

[43] A. Astaras, A. Athanasiou, A. Alexandrou, P. Kartsidis, N. Moustakas, and P. D. Bamidis, "Double-blind greek translation 
and online implementation of the Godspeed robotics questionnaire," in Proceedings of the in 6th Panhellenic Conference on Biomedical Technology Conference, p. 34, 2015.

[44] E. I. Konstantinidis, A. Billis, C. Bratsas, A. Siountas, and P. D. Bamidis, "Thessaloniki active and healthy ageing living lab: The roadmap from a specific project to a living lab towards openness," in Proceedings of the 9th ACM International Conference on PErvasive Technologies Related to Assistive Environments (PETRA '2016), pp. 1-4, July 2016.

[45] E. I. Konstantinidis and P. D. Bamidis, "Density based clustering on indoor kinect location tracking: A new way to exploit active and healthy aging living lab datasets," in Proceedings of the 2015 IEEE 15th International Conference on Bioinformatics and Bioengineering (BIBE), pp. 1-6, November 2015.

[46] E. I. Konstantinidis, P. E. Antoniou, G. Bamparopoulos, and P. D. Bamidis, "A lightweight framework for transparent cross platform communication of controller data in ambient assisted living environments," Information Sciences, vol. 300, no. 1, pp. 124-139, 2015.

[47] T. Sollfrank, D. Hart, R. Goodsell, J. Foster, and T. Tan, “3D visualization of movements can amplify motor cortex activation during subsequent motor imagery," Frontiers in Human Neuroscience, vol. 9, article 463, 2015.

[48] D. Cramer, "Fundamental statistics for social research," in Stepby-step calculations and computer techniques using SPSS for Windows, Routledge, London, UK, 1988.

[49] D. Cramer and D. Howitt, The SAGE Dictionary of Statistics, SAGE Publications, 2004.

[50] D. P. Doane and L. E. Seward, "Measuring skewness: a forgotten statistic?" Journal of Statistics Education, vol. 19, no. 2, pp. 1-18, 2011.

[51] N. M. Razali, Y. B. Wah, and M. Sciences, "Power comparisons of Shapiro-Wilk, Kolmogorov-Smirnov," Lilliefors and Anderson-Darling tests, vol. 2, no. 1, pp. 21-33, 2011.

[52] S. S. Shapiro and M. B. Wilk, "An analysis of variance test for normality: Complete samples," Biometrika, vol. 52, pp. 591-611, 1965.

[53] N. Mogey, "So You Want to Use a Likert Scale?" in Edinburgh: 2 Evaluation Cookbook Learning Technology Dissemination Initiative, Institute for Computer Based Learning, Heriot-Watt University, 1998.

[54] N. Birbaumer and P. Sauseng, "Brain-Computer Interface in Neurorehabilitation," 2010.

[55] A. Vuckovic, J. A. Pineda, K. LaMarca, D. Gupta, and C. Guger, "Interaction of BCI with the underlying neurological conditions in patients: pros and cons," Frontiers in Neuroengineering, vol. 7, 2014.

[56] F. Nijboer, N. Birbaumer, and A. Kübler, "The influence of psychological state and motivation on brain-computer interface performance in patients with amyotrophic lateral sclerosis - a longitudinal study," Frontiers in Neuroscience, vol. 4, article no. $55,2010$.

[57] E. M. Hammer, S. Halder, B. Blankertz et al., "Psychological predictors of SMR-BCI performance," Biological Psychology, vol. 89, no. 1, pp. 80-86, 2012.

[58] C. Papadelis, C. Kourtidou-Papadeli, P. Bamidis, and M. Albani, "Effects of imagery training on cognitive performance and use of physiological measures as an assessment tool of mental effort," Brain and Cognition, vol. 64, no. 1, pp. 74-85, 2007.

[59] F. González-Palau, M. Franco, P. D. Bamidis et al., “The effects of a computer-based cognitive and physical training program in a healthy and mildly cognitive impaired aging sample," Aging \& Mental Health, vol. 18, no. 7, pp. 838-846, 2014.

[60] S. M. Roldan, “Object Recognition in Mental Representations: Directions for Exploring Diagnostic Features through Visual Mental Imagery," Frontiers in Psychology, vol. 8, 2017.

[61] E. Broadbent, "Interactions with Robots: The Truths We Reveal about Ourselves," Annual Review of Psychology, vol. 68, pp. 627652, 2017.

[62] E. López-Larraz, F. Trincado-Alonso, V. Rajasekaran et al., "Control of an ambulatory exoskeleton with a brain-machine interface for spinal cord injury gait rehabilitation," Frontiers in Neuroscience, vol. 10, 2016.

[63] G. E. Francisco, N. Yozbatiran, J. Berliner et al., "Robot-Assisted Training of Arm and Hand Movement Shows Functional Improvements for Incomplete Cervical Spinal Cord Injury," American Journal of Physical Medicine \& Rehabilitation, vol. 96, pp. S171-S177, 2017.

[64] A. Jackson and J. B. Zimmermann, "Neural interfaces for the brain and spinal cord - Restoring motor function," Nature Reviews Neurology, vol. 8, no. 12, pp. 690-699, 2012.

[65] R. Grech, T. Cassar, J. Muscat et al., "Review on solving the inverse problem in EEG source analysis," Journal of NeuroEngineering and Rehabilitation, vol. 5, article 25, 2008.

[66] S. Baillet, J. C. Mosher, and R. M. Leahy, "Electromagnetic brain mapping," IEEE Signal Processing Magazine, vol. 18, no. 6, pp. 14-30, 2001.

[67] C. J. Holmes, R. Hoge, L. Collins, R. Woods, A. W. Toga, and A. C. Evans, "Enhancement of MR images using registration for signal averaging," Journal of Computer Assisted Tomography, vol. 22, no. 2, pp. 324-333, 1998.

[68] B. J. Edelman, B. Baxter, and B. He, "EEG source imaging enhances the decoding of complex right-hand motor imagery tasks,' IEEE Transactions on Biomedical Engineering, vol. 63, no. 1, pp. 4-14, 2016.

[69] H. Yuan, T. Liu, R. Szarkowski, C. Rios, J. Ashe, and B. He, "Negative covariation between task-related responses in alpha/betaband activity and BOLD in human sensorimotor cortex: an EEG and fMRI study of motor imagery and movements," NeuroImage, vol. 49, no. 3, pp. 2596-2606, 2010.

[70] B. Kamousi, A. N. Amini, and B. He, "Classification of motor imagery by means of cortical current density estimation and Von Neumann entropy," Journal of Neural Engineering, vol. 4, no. 2, article no. 002, pp. 17-25, 2007.

[71] H. Ramoser, J. Müller-Gerking, and G. Pfurtscheller, “Optimal spatial filtering of single trial EEG during imagined hand movement," IEEE Transactions on Neural Systems and Rehabilitation Engineering, vol. 8, no. 4, pp. 441-446, 2000.

[72] G. R. Müller-Putz, I. Daly, and V. Kaiser, "Motor imageryinduced EEG patterns in individuals with spinal cord injury and their impact on brain-computer interface accuracy," Journal of Neural Engineering, vol. 11, no. 3, Article ID 035011, 2014.

[73] G. Onose, C. Grozea, A. Anghelescu et al., "On the feasibility of using motor imagery EEG-based brain-computer interface in chronic tetraplegics for assistive robotic arm control: A clinical test and long-term post-trial follow-up," Spinal Cord, vol. 50, no. 8, pp. 599-608, 2012. 


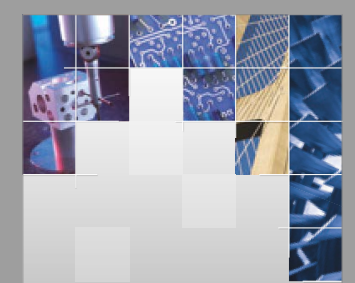

\section{Enfincering}
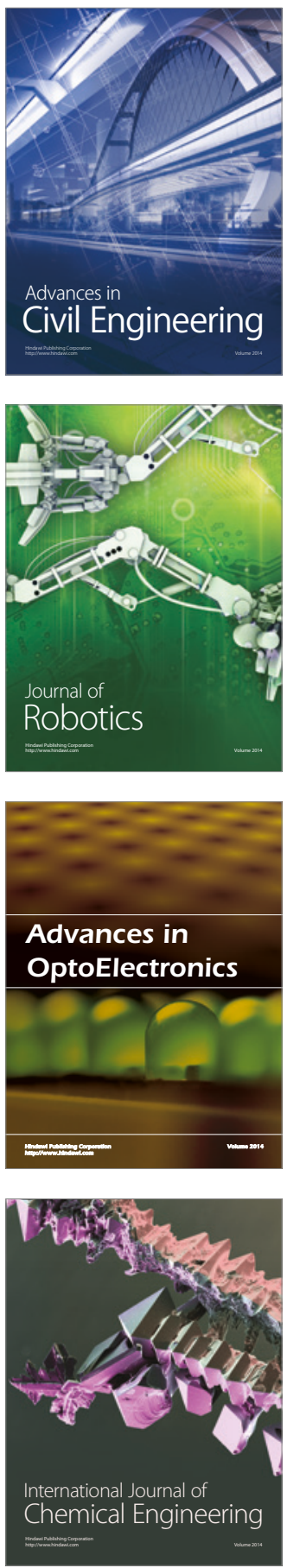

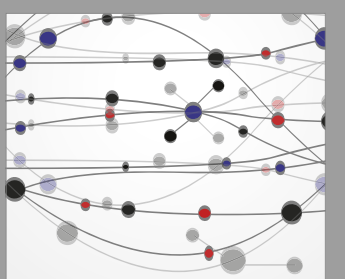

The Scientific World Journal

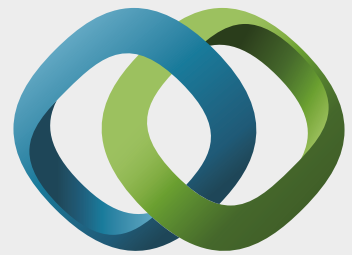

\section{Hindawi}

Submit your manuscripts at

https://www.hindawi.com
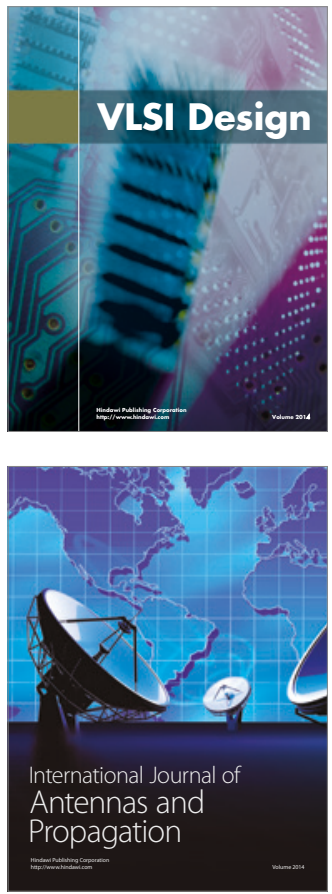

\section{Rotating}

Machinery
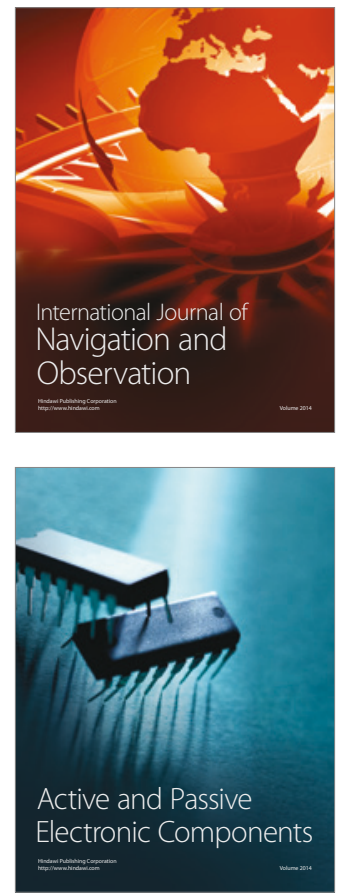
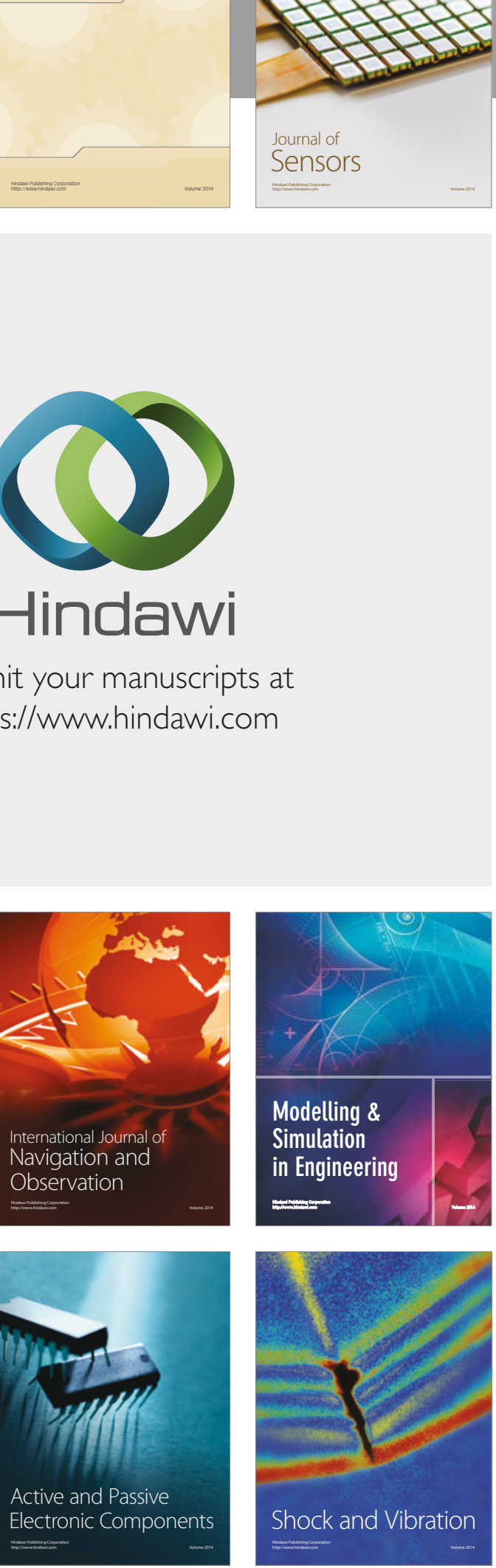
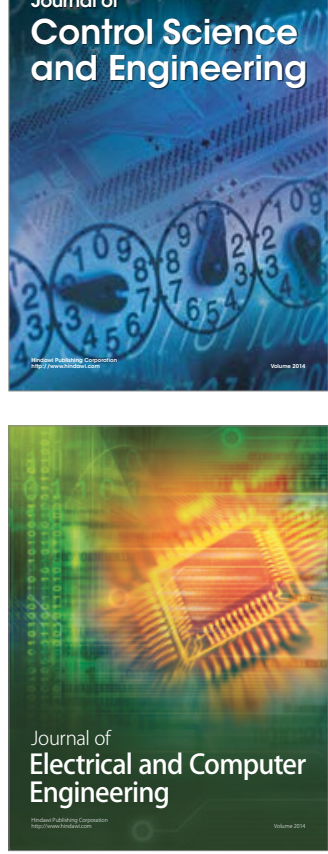

Distributed

Journal of

Control Science

and Engineering
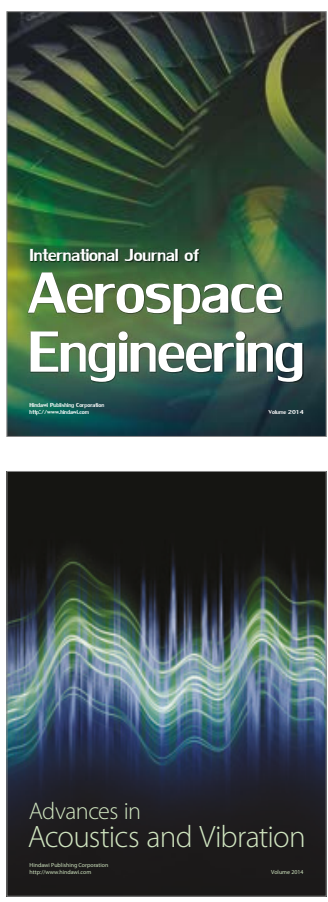

Sensor Networks 